\title{
Review
}

\section{Classification of oesophageal motility abnormalities}

\begin{abstract}
Summary
Manometric examination of the oesophagus frequently reveals abnormalities whose cause is unknown and whose physiological importance is not clear. A large body of literature dealing with oesophageal motility abnormalities has evolved over the past few decades but comparisons among studies have been compromised by the lack of a widely accepted system for classifying the abnormal motility patterns, and by the lack of uniform diagnostic criteria for the putative disorders. Based on an extensive review and analysis of the literature, this report suggests an operational scheme to be used for the general classification of oesophageal motility abnormalities, and proposes standardised manometric criteria for the putative oesophageal motility disorders. By applying the guidelines proposed in this report, clinicians and researchers can determine if their patients fulfil the manometric criteria for a putative motility disorder. This should facilitate and improve comparisons among patients and studies. However, it is important to emphasise that fulfilment of the proposed criteria does not establish the clinical importance of the motility abnormalities.
\end{abstract}

\section{Introduction}

Oesophageal motility studies are performed in patients who have dysphagia that is not explained by stenoses or inflammation of the oesophagus, and for patients who have chest pain that is not explained by heart disease or other thoracic disorders. ${ }^{1}$ Although manometric evaluations of such patients often reveal abnormalities, ${ }^{2}$ authorities frequently disagree on how to interpret and classify the aberrant oesophageal motility patterns. ${ }^{3}$ Attempts to devise a meaningful classification system for oesophageal motility abnormalities have been confounded by several factors. Firstly, the cause of most oesophageal motility abnormalities is not known. ${ }^{4}$ This precludes an ideal classification that would be based on an understanding of the fundamental pathophysiology. Next, a number of manometric abnormalities have no apparent physiological consequences. For example, the high amplitude peristaltic waves that characterise the so-called "nutcracker oesophagus" may not interfere with oesophageal clearance, may not cause demonstrable abnormalities on barium contrast examinations, and may not correlate with episodes of dysphagia or chest pain. ${ }^{5}$ Furthermore, symptoms may not respond to therapies that correct the manometric abnormalities. For example, patients who have chest pain and high amplitude peristaltic waves in the oesophagus often report no relief of pain during treatment with calcium channel blockers that cause significant reductions in wave amplitudes. ${ }^{6}$ Hence it is not clear that certain "oesophageal motility disorders" are in fact disorders. In some cases the manometric abnormalities observed may be merely epiphenomena - that is, effects or associations rather than causes of the primary problems. Finally, there may be no extramanometric means to validate the diagnosis of putative disorders that have been defined solely on the basis of manometric features, such as nutcracker oesophagus.

Although the clinical importance of certain manometric abnormalities remains disputed, a large body of literature dealing with putative oesophageal motility disorders has evolved over the past few decades. ${ }^{7}$ Comparisons between studies are often compromised by the fact that different groups of investigators have used different manometric criteria to identify the same putative disorder. The purpose of this report is to suggest a general operational scheme for the classification of oesophageal motility abnormalities and to propose standardised manometric criteria for the abnormal oesophageal motility patterns. The proposed criteria are based on an extensive review and analysis of clinical reports on oesophageal motility abnormalities published in peer reviewed journals since 1966. Reports reviewed were identified primarily by a Medline search using the $\mathrm{MeSH}$ term search strategy "esophageal motility disorders and manometry and diagnosis". Studies that focused predominantly on motility abnormalities in gastro-oesophageal reflux disease were largely excluded. Inconsistencies in diagnostic criteria among the reports are noted, and rationales are provided for the standardised diagnostic criteria proposed.

Application of uniform criteria for the study of oesophageal motility abnormalities is an important step towards determining their clinical significance. However, it is important to emphasise that fulfilment of the manometric criteria for a putative motility disorder does not establish the clinical importance of the motility abnormality, nor does it establish that the manometric phenomena are manifestations of a disease process. The ultimate diagnosis of a motility disorder requires consideration of clinical as well as manometric data. By applying the guidelines proposed in this report, the clinician can determine only whether a patient fulfils the manometric, not the clinical criteria for a given motility abnormality.

\section{General classification of oesophageal manometric abnormalities}

The four major patterns of oesophageal manometric abnormalities are summarised in table 1 . Processes that affect the inhibitory innervation of the lower oesophageal sphincter (LOS) can cause inadequate LOS relaxation (relaxation that is incomplete in amplitude or too short in duration). ${ }^{78}$ In the body of the oesophagus, abnormal motility is manifest as uncoordinated contraction, hypercontraction, and hypocontraction. ${ }^{9}$ For patients who have motility problems that cause both inadequate LOS relaxation and oesophageal body dysfunction (for example, classic achalasia), symptoms are often caused primarily by the abnormality in LOS relaxation. Inadequate LOS relaxation delays oesophageal clearance, and treatments for patients with inadequate LOS relaxation focus on reducing LOS pressure through pharmacological or mechanical means. Unlike some abnormal motility patterns, the finding of inadequate LOS relaxation has both physiological importance and therapeutic implications. Therefore, symptomatic patients with inadequate LOS relaxation clearly have a clinical disorder. For these reasons, patients who exhibit this abnormality can be categorised as having a disorder of inadequate LOS relaxation, regardless of the

Abbreviations used in this paper: LOS, lower oesophageal sphincter; UOS, upper oesophageal sphincter. 
Table 1 Classification of oesophageal motility abnormalities

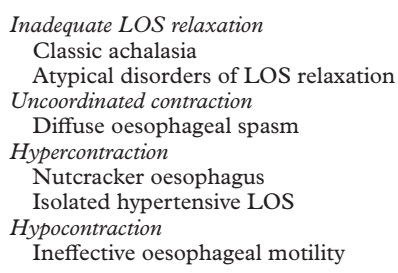

motility pattern observed in the body of the oesophagus. Classic achalasia is included in this category of inadequate LOS relaxation, as are a number of other disorders that do not exhibit the abnormalities in oesophageal body function that are characteristic of classic achalasia.

Contractions in the body of the oesophagus that are not peristaltic and aborally directed (for example, simultaneous, retrograde, segmental contractions) can be considered uncoordinated. Uncoordinated oesophageal contractions can interfere with oesophageal clearance. ${ }^{10}$ Diffuse oesophageal spasm is a condition characterised primarily by uncoordinated motility in the body of the oesophagus.

Hypercontraction abnormalities are those characterised by oesophageal muscle contractions that are of high amplitude, long duration, or both. The putative disorders of hypercontraction (for example, nutcracker oesophagus, isolated hypertensive LOS) are perhaps the most controversial of the abnormal oesophageal motility patterns because it is not clear that oesophageal hypercontraction has any physiological importance. In contrast, hypocontraction abnormalities that result from weak (low amplitude) muscle contractions can cause ineffective oesophageal motility that delays oesophageal clearance, and LOS hypotension that can result in gastro-oesophageal reflux.

Most oesophageal motility abnormalities fall predominantly into one of the four major categories shown in table 1 although there can be considerable overlap with some conditions exhibiting features of more than one category. Nevertheless, this classification has clinical implications that may be helpful in patient management. For example, patients with disorders characterised by inadequate LOS relaxation often benefit from pharmacological or mechanical therapies aimed at reducing LOS pressure. Also, patients with hypocontraction abnormalities may need treatment for gastro-oesophageal reflux disease and may benefit from prokinetic agents.

\section{The oesophageal manometric procedure}

There is no clear consensus on the ideal technique for performing oesophageal manometry. To use the general classification system for oesophageal motility abnormalities proposed above, the manometry must at least provide an assessment of the completeness of LOS relaxation and an evaluation of peristaltic function of the oesophageal body. This can be accomplished as follows ${ }^{11}$ : the motility catheter is passed through the nose or mouth until the pressure sensors are positioned in the stomach, and gastric baseline pressure is recorded. While the patient breathes quietly, a slow, station pull through is performed for evaluation of LOS pressure. LOS pressure is measured as the mid respiratory level in the area of maximal pressure (compared with gastric baseline pressure). Although it is conventional to refer to this measured value as "LOS pressure", the value reflects pressure generated by the crural diaphragm as well as the LOS muscle. ${ }^{12}$ With the pressure sensor positioned in the LOS, the patient is asked to perform at least five separate wet swallows ( $5 \mathrm{ml}$ of water) to assess the completeness of swallow induced LOS relaxation. Completeness of relaxation is determined by measuring the
Table 2 Normal oesophageal manometric features

\begin{tabular}{ll}
\hline Basal LOS pressure & $\begin{array}{l}10-45 \mathrm{~mm} \mathrm{Hg} \text { (mid respiratory pressure } \\
\text { measured by station pull through } \\
\text { technique) }\end{array}$ \\
LOS relaxation with swallow & $\begin{array}{l}\text { Complete (to a level }<8 \mathrm{~mm} \mathrm{Hg} \text { above } \\
\text { gastric pressure) }\end{array}$ \\
Wave progression & $\begin{array}{l}\text { Peristalsis progressing from UOS through } \\
\text { LOS at a rate of } 2-8 \mathrm{~cm} / \mathrm{s}\end{array}$ \\
Distal wave amplitude & $\begin{array}{l}30-180 \mathrm{~mm} \mathrm{Hg} \text { (average of } 10 \text { swallows at } \\
\text { two recording sites positioned } 3 \text { and } 8 \mathrm{~cm} \\
\text { above the LOS) }\end{array}$
\end{tabular}

LOS, lower oesophageal sphincter; UOS, upper oesophageal sphincter.

residual LOS pressure - that is, the difference between the pressure recorded at the nadir of LOS relaxation and gastric baseline pressure. Next, peristalsis is evaluated by positioning at least three pressure sensors separated by intervals of $5 \mathrm{~cm}$ in the body of the oesophagus. The distal sensor is positioned at a level $3 \mathrm{~cm}$ above the LOS, and a series of 10 wet swallows is performed. The swallows are separated by an interval of at least 30 seconds, and pressure wave amplitude, duration, and velocity are measured.

Normal values for oesophageal manometry are provided in table 2 . These values were derived from a study that used the manometry protocol described above in 95 healthy adult volunteers. ${ }^{13}$ The range of normal values listed comprises two standard deviations (SD) above and below the means.

\section{Conditions with inadequate LOS relaxation}

CLASSIC ACHALASIA

Of all the proposed oesophageal motility disorders, classic achalasia is perhaps the best understood and best characterised. Classic achalasia is an oesophageal disease of unknown cause in which there is degeneration of neurones in the wall of the oesophagus. ${ }^{14} 15$ The degenerative process appears to involve preferentially the nitric oxide producing inhibitory neurones that effect relaxation of oesophageal smooth muscle. ${ }^{16}{ }^{17}$ The smooth muscle of the LOS is normally tonically contracted at rest and relaxes when intramural neurones release their inhibitory neurotransmitters. ${ }^{18}$ Loss of inhibitory innervation in the LOS can cause basal sphincter pressures to rise and can interfere with normal relaxation. Unlike the LOS, the smooth muscle of the oesophageal body does not exhibit resting tone and therefore loss of inhibitory neurones has little effect on resting pressure in the body of the oesophagus. Inhibitory influences are necessary for normal peristalsis however and loss of inhibitory neurones can result in aperistalsis. ${ }^{19}$ In classic achalasia, swallows are followed either by no discernible activity in the oesophageal body or by simultaneous oesophageal contractions of low amplitude (<40 mm Hg). ${ }^{20}$

Although the aetiology of primary achalasia is not known, certain recognised diseases can cause oesophageal motor abnormalities similar or identical to those of primary achalasia. In Chagas disease seen in Central and South America, for example, oesophageal infection with the protozoan parasite Trypanosoma cruzi can result in loss of intramural ganglion cells that causes aperistalsis and incomplete LOS relaxation. ${ }^{21}$ Malignancies can also cause an achalasia-like disorder (pseudoachalasia) either by invading the oesophageal neural plexuses directly (for example, adenocarcinoma of the oesophagogastric junction) or through release of uncharacterised humoral factors that disrupt oesophageal function as part of a paraneoplastic syndrome. ${ }^{22}$ Primary and secondary achalasia cannot be distinguished reliably on the basis of manometric criteria alone.

Achalasia is a Greek term that means "does not relax". Although many abnormalities in oesophageal body function can be observed in patients with achalasia, symptoms 

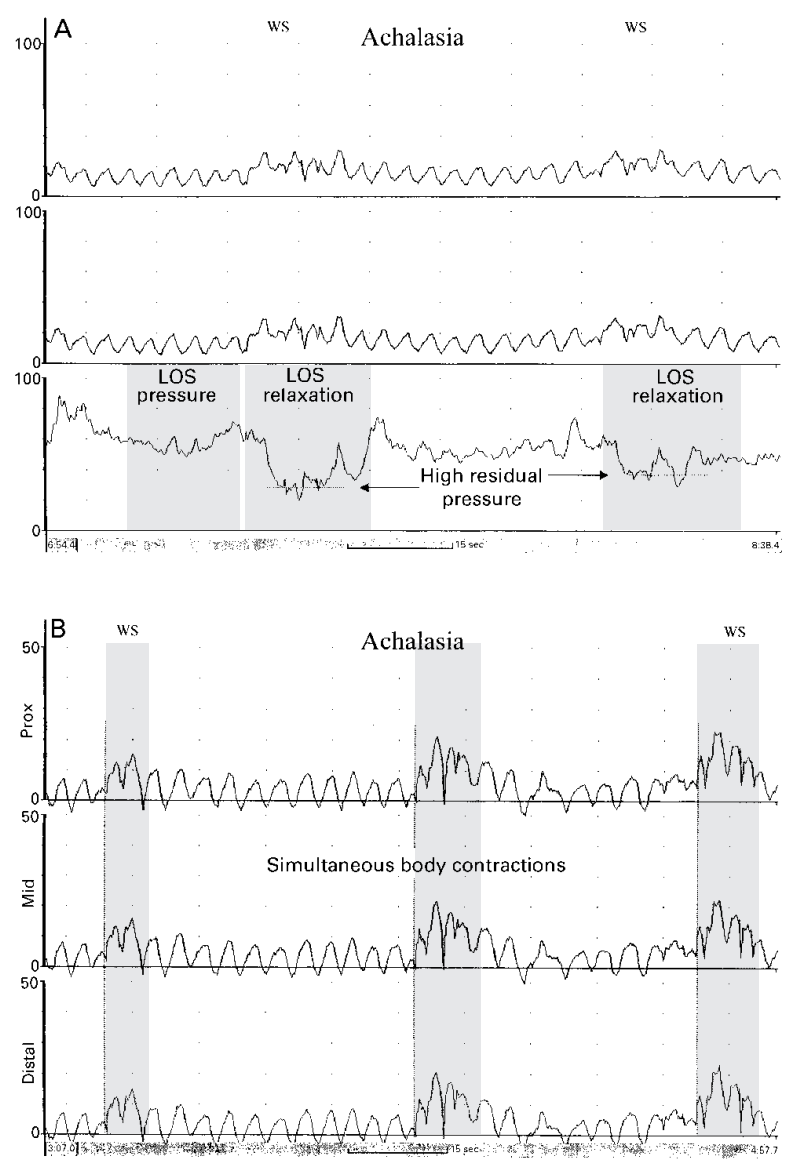

Figure 1 (A) Oesophageal manometry tracing from a patient with classic achalasia. The distal recording site, positioned in the lower oesophageal sphincter (LOS), shows high basal LOS pressure (approximately $60 \mathrm{~mm} \mathrm{Hg}$ ). Note that the two wet swallows (WS) are followed by incomplete relaxation of the LOS with residual pressure values of 24 and $36 \mathrm{~mm} \mathrm{Hg}$. The two proximal recording sites, located 3 and 8 $\mathrm{cm}$ above the LOS, show that wet swallows are not attended by peristalsis. (B) Oesophageal manometry tracing from a patient with classic achalasia. The three recording sites are positioned 3, 8, and $13 \mathrm{~cm}$ above the LOS. Note that wet swallows (WS) are not followed by peristaltic contractions, and that the pressure changes recorded in the oesophageal body are simultaneous, low amplitude, and identical in appearance (isobaric).

are due primarily to the fact that swallow induced LOS relaxation is inadequate either because it is incomplete in amplitude or too short in duration. ${ }^{23}$ Classic achalasia is a well described clinical entity but a number of atypical disorders of LOS relaxation have been reported that have manometric features not characteristic of classic achalasia (for example, preserved peristalsis, apparent complete LOS relaxation). ${ }^{24-26}$ The clinical feature common to all of these atypical disorders is that affected patients have dysphagia which responds to treatments that decrease resting LOS pressure (for example, pneumatic dilation, Heller myotomy, botulinum toxin injection). Thus the atypical disorders of LOS relaxation are not defined adequately by manometric criteria alone. There are clinical and experimental data to suggest that some of the atypical disorders of LOS relaxation may be early manifestations of the same enteric neuropathy that causes classic achalasia, and that these atypical disorders eventually may evolve into classic achalasia. ${ }^{24} 25$

The manometric features proposed for a diagnosis of classic achalasia are: (1) incomplete relaxation of the LOS (defined as a mean swallow induced fall in resting LOS pressure to a nadir value $>8 \mathrm{~mm}$ above gastric pressure) (fig 1A) and (2) aperistalsis in the body of the oesophagus

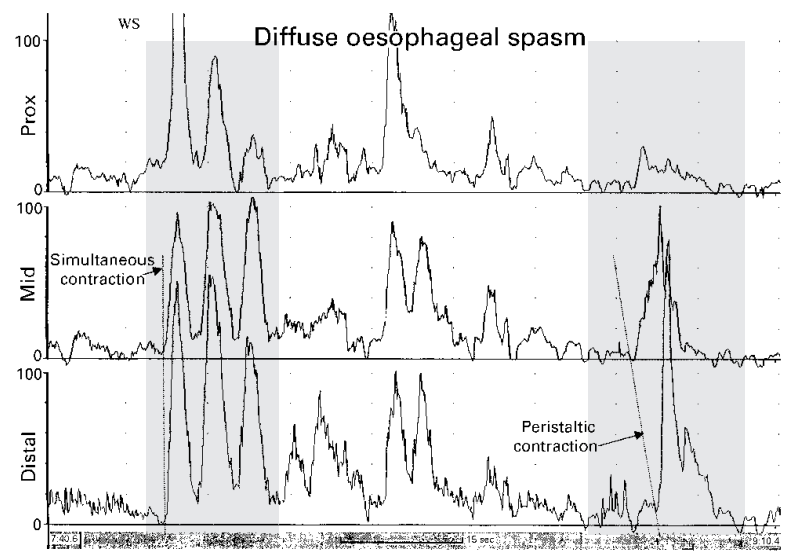

Figure 2 Oesophageal manometry tracing from a patient with diffuse oesophageal spasm. The recording sites are positioned 3, 8 , and $13 \mathrm{~cm}$ above the lower oesophageal sphincter. Note that the first wet swallow (WS) is followed by oesophageal contractions that are simultaneous and repetitive. However, some peristaltic activity is preserved, as evidenced by the peristaltic contraction of the oesophageal body shown in the sequence on the right.

characterised either by simultaneous oesophageal contractions with amplitudes $<40 \mathrm{~mm} \mathrm{Hg}$ or by no apparent oesophageal contractions (fig 1A, 1B). Manometric features that are characteristic of classic achalasia but not required for the diagnosis include: (1) elevated resting LOS pressure ( $>45 \mathrm{~mm} \mathrm{Hg}$ ) (fig $1 \mathrm{~A}$ ) and (2) resting pressure in the oesophageal body that exceeds resting pressure in the stomach. A number of abnormalities in upper oesophageal sphincter (UOS) function also have been described in achalasia including: (1) elevated UOS residual pressure, ${ }^{27}$ (2) decreased duration of UOS relaxation, ${ }^{18}$ (3) repetitive UOS contractions, ${ }^{28}$ and (4) an abnormal belch reflex. ${ }^{29}$ These UOS abnormalities are not evaluated routinely on oesophageal manometric evaluation and none is required to establish the diagnosis of classic achalasia.

ATYPICAL DISORDERS OF LOS RELAXATION

Patients with atypical disorders of LOS relaxation have one or more manometric features that preclude a diagnosis of classic achalasia, including: (1) some preserved peristalsis, (2) oesophageal contractions with amplitudes $>40 \mathrm{~mm} \mathrm{Hg}$, and (3) complete LOS relaxation of inadequate duration. ${ }^{20-26}$ These patients have dysphagia, usually for liquids as well as solids, that is not explained by fixed stenoses or inflammation in the oesophagus. The barium swallow often, but not invariably, shows features suggestive of classic achalasia with a dilated oesophagus that terminates in a beak-like narrowing caused by the dysfunctional LOS. Although evidence of inadequate LOS relaxation is found in all cases, the inadequacy may not be apparent by standard manometric analyses. For example, some patients exhibit LOS relaxation that is complete in amplitude but inadequate in duration (that is, the LOS contracts before it is traversed by the peristaltic wave).${ }^{26}$ Duration of LOS relaxation is not reported routinely by most motility laboratories. Although the atypical disorders of LOS relaxation can be suspected on the basis of symptoms and manometric features, confirmation of the diagnosis ultimately requires relief of dysphagia by a treatment that decreases resting LOS pressure such as calcium channel blockers, pneumatic dilation, Heller myotomy, or botulinum toxin injection.

It is not clear that identification of the atypical manometric features has any specific clinical or therapeutic implications. Some investigators have proposed that chest pain may be more prominent in patients with a variant of achalasia that is characterised by oesophageal contractions 
with amplitudes $>40 \mathrm{~mm} \mathrm{Hg}$ (so-called "vigorous achalasia") whereas others have found that such patients cannot be distinguished clinically from those with classic achalasia. ${ }^{20}$ One group has reported that injection of botulinum toxin is more effective for treating vigorous than classic achalasia $^{30}$ whereas another group found no such difference in the response to toxin injection. ${ }^{31}$ Rather than assigning names of dubious significance (for example, vigorous achalasia) to the atypical disorders of LOS relaxation, it seems preferable to use descriptive terms (for example, inadequate LOS relaxation with preserved peristalsis or inadequate LOS relaxation with preserved peristalsis and oesophageal contractions with amplitudes $>40 \mathrm{~mm} \mathrm{Hg}$, etc.).

\section{Uncoordinated oesophageal contraction}

\section{DIFFUSE OESOPHAGEAL SPASM}

Diffuse oesophageal spasm is a condition of unknown aetiology that is manifested clinically by episodes of dysphagia and chest pain, radiographically by tertiary contractions of the oesophagus, and manometrically by uncoordinated ("spastic") activity in the smooth muscle portion of the oesophagus. ${ }^{32}$ The pathophysiology and natural history of the disorder are poorly understood. Furthermore, authorities disagree on precisely how spastic activity manifests itself manometrically. Comparisons between studies on diffuse oesophageal spasm have been compromised by the lack of universally accepted diagnostic criteria for the condition. Different investigators have used different diagnostic criteria and, consequently, it is likely that patients with a number of different disorders have been included in studies on diffuse oesophageal spasm. In the absence of a diagnostic gold standard for a disorder, conclusions regarding the validity of any diagnostic test are suspect.

Published manometric criteria for diffuse oesophageal spasm have differed regarding the requirement for the presence of spontaneous and repetitive contractions, and with regard to the need to demonstrate oesophageal contractions that are of high amplitude and prolonged duration. However, simultaneous oesophageal contractions have been found in the majority of patients in published reports. In 1984, Richter and Castell called for a reappraisal of the diagnostic criteria for diffuse oesophageal spasm based on their experience and review of published studies. ${ }^{32}$ They pointed out the disparities in the published series and emphasised the importance of simultaneous contractions induced by wet swallows as the key diagnostic criterion for diffuse oesophageal spasm. These investigators have contended that diffuse oesophageal spasm is an uncommon condition that is defined manometrically when $>10 \%$ (but $<100 \%$ ) of wet swallows are followed by simultaneous oesophageal contractions, regardless of contraction amplitude and duration. ${ }^{33}$ They have argued that diffuse oesophageal spasm would be an extraordinarily rare condition if high amplitude simultaneous contractions were required for the diagnosis.

An issue that arises in defining diffuse oesophageal spasm primarily by the presence of simultaneous contractions is that such contractions can be found in patients with a variety of recognised disorders, including diabetes mellitus, alcoholism, amyloidosis, and scleroderma, as well as in patients who have gastro-oesophageal reflux disease that is not associated with other diseases. ${ }^{34}$ This situation is similar to that described above for achalasia in which motility abnormalities identical to those of primary achalasia can be seen in other disorders, such as Chagas disease and gastric cancer. Unlike the situation for achalasia however it is not common practice to categorise diffuse oesophageal spasm as primary or secondary in nature. Furthermore, the physiological and clinical consequences of the low amplitude simultaneous contractions seen in scleroderma may differ substantially from those of the normal or high amplitude simultaneous contractions seen in patients with idiopathic diffuse oesophageal spasm. Consequently, it may be inappropriate to include patients with low amplitude simultaneous contractions under the rubric of "diffuse oesophageal spasm". ${ }^{35}$

Another problem in identifying diffuse oesophageal spasm primarily by the presence of simultaneous oesophageal contractions is that, in some cases, it may be difficult to distinguish diffuse oesophageal spasm from the atypical disorders of LOS relaxation. In addition to inadequate LOS relaxation, simultaneous oesophageal contractions are a hallmark of primary achalasia. Inadequate LOS relaxation has been described in diffuse oesophageal spasm, and the only feature used to distinguish these two disorders manometrically is the arbitrary requirement that some normal peristalsis be preserved in diffuse oesophageal spasm. ${ }^{33}$ For a patient who has the clinical and radiographic features of classic achalasia, whose manometric examination shows inadequate LOS relaxation and simultaneous oesophageal contractions, and whose dysphagia resolves with pneumatic dilation, it seems inappropriate to call the disorder diffuse oesophageal spasm simply because there are occasional normal peristaltic sequences. This constellation of findings is better characterised as an atypical disorder of LOS relaxation.

The manometric features proposed for a diagnosis of diffuse oesophageal spasm are: (1) simultaneous contractions associated with $>10 \%$ of wet swallows and (2) mean simultaneous contraction amplitude $>30 \mathrm{~mm} \mathrm{Hg}$ (fig 2). Features that can be found commonly but are not required for manometric diagnosis include: (1) spontaneous contractions, (2) repetitive contractions, (3) multiple peaked contractions, and (4) intermittent normal peristalsis. If there is incomplete relaxation of the LOS (defined as a mean swallow induced fall in resting LOS pressure to a nadir value $>8 \mathrm{~mm}$ above gastric pressure), the condition is better classified as an atypical disorder of LOS relaxation.

\section{Oesophageal hypercontraction}

\section{NUTCRACKER OESOPHAGUS}

"Nutcracker oesophagus" is a term coined by Castell and colleagues for the condition in which patients with non-cardiac chest pain and/or dysphagia exhibit peristaltic waves in the distal oesophagus with mean amplitudes exceeding normal values by more than 2 SD. ${ }^{36}$ Although high amplitude peristaltic sequences are the most common motility abnormalities observed in patients with noncardiac chest pain, ${ }^{2}$ the clinical and physiological importance of these sequences is disputed. ${ }^{3-6}$ Areas of disparity among published reports regarding the manometric criteria for nutcracker oesophagus have included the precise amplitude of the peristaltic waves required for the diagnosis, the precise oesophageal levels at which the wave amplitudes have been measured, the importance of segmental (as opposed to diffuse) high amplitude contractions, the prevalence of peristaltic waves of abnormally long duration, and the prevalence of abnormalities in LOS function.

Whereas the nutcracker oesophagus has been defined by the presence of peristaltic waves with mean amplitudes $>2$ SD above normal, the precise amplitude required for the diagnosis can vary depending on the data used to establish normal values. In the initial report on the condition by Benjamin et al published in 1979, the mean distal oesophageal peristaltic wave amplitude in 40 healthy subjects was 81 (15) $\mathrm{mm} \mathrm{Hg}$ (1 SD). ${ }^{37}$ The authors suggested a cut off value of $120 \mathrm{~mm} \mathrm{Hg}$ (slightly more than $2 \mathrm{SD}$ above the mean value) as a diagnostic criterion for high amplitude 


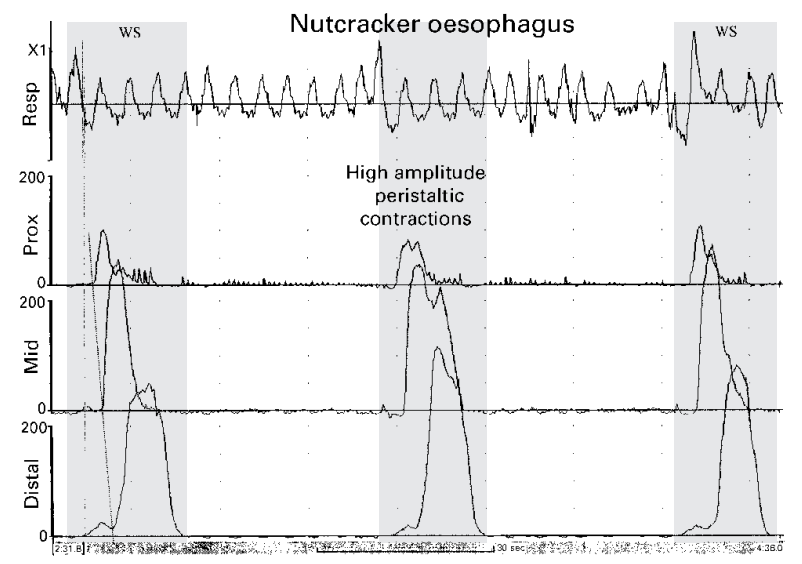

Figure 3 Oesophageal manometry tracing from a patient with nutcracker oesophagus. The recording sites are positioned 3, 8, and $13 \mathrm{~cm}$ above the lower oesophageal sphincter. Note the high amplitude peristaltic contractions initiated by wet swallows (WS).

peristalsis. As mentioned, a subsequent study of 95 healthy adults found that the mean amplitude of peristaltic waves

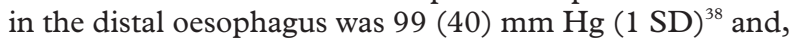
based on this report, a number of subsequent reports on nutcracker oesophagus have used $180 \mathrm{~mm} \mathrm{Hg}$ as the diagnostic criterion. ${ }^{34}$

Peristaltic wave amplitude varies considerably throughout the oesophagus, both in normal individuals and in patients with nutcracker oesophagus. ${ }^{13} 38$ A number of studies on the condition have used the average value of peristaltic wave amplitudes measured at two distal recording sites to determine mean distal oesophageal peristaltic wave amplitude. Some investigators have used recording sites positioned 3 and $8 \mathrm{~cm}$ above the $\operatorname{LOS}^{36} 39$ whereas others have used sites positioned 2 and $7 \mathrm{~cm}$ above the LOS for measurement of mean distal oesophageal peristaltic wave pressure. ${ }^{40}$ Some studies have shown that high amplitude contractions may involve the oesophagus in a segmental fashion, with high amplitude peristaltic waves found in only one of the two recording sites in the distal oesophagus (for example, at $3 \mathrm{~cm}$ but not at $8 \mathrm{~cm}$ above the LOS or vice versa). ${ }^{39} 40$ The importance of these segmental high amplitude contractions is not clear.

In the aforementioned manometric study of 95 healthy adults, mean duration of peristaltic waves in the distal oesophagus was $3.9(0.9)$ seconds ( 1 SD).${ }^{13}$ Based on this report, a contraction duration of $>6$ seconds (slightly more than $2 \mathrm{SD}$ above the mean value) can be regarded as abnormal. Many patients with nutcracker oesophagus exhibit peristaltic contractions with durations that exceed 6 seconds. ${ }^{34}$ Some investigators have even described a group of patients with chest pain associated with peristaltic contractions of long duration but normal amplitude in the distal oesophagus. ${ }^{41}$ No studies have required prolonged peristaltic contraction duration as a diagnostic criterion for nutcracker oesophagus, and the physiological and clinical consequences of such contractions are not clear. Finally, high resting pressures in the LOS have been found in some patients with nutcracker oesophagus. ${ }^{34}$

The manometric feature proposed for a diagnosis of nutcracker oesophagus is a mean distal oesophageal peristaltic wave amplitude $>180 \mathrm{~mm} \mathrm{Hg}$ (measured as the average amplitude of 10 swallows at two recording sites positioned 3 and $8 \mathrm{~cm}$ above the LOS) (fig 3). Peristaltic contractions of long duration are found commonly but are not required for manometric diagnosis of nutcracker oesophagus. Resting pressure in the LOS is usually normal but may be elevated in which case patients are categorised as having nutcracker oesophagus with a hypertensive LOS.
ISOLATED HYPERTENSIVE LOS

Although the hypertensive LOS was described almost 40 years ago, ${ }^{42}$ it remains unclear whether this condition per se has any clinical or physiological consequences. Published studies on the hypertensive LOS have included patients with a normal progression of peristalsis in the distal oesophagus, and with any or all combinations of three LOS abnormalities: (1) abnormally elevated resting LOS pressure (>2 SD above the normal mean value), ${ }^{43}$ (2) exaggerated contraction of the LOS after relaxation (after contraction), ${ }^{44}$ and (3) incomplete LOS relaxation. ${ }^{45}$ It is not clear how or even if the former two LOS abnormalities cause symptoms. Incomplete LOS relaxation however clearly can interfere with oesophageal clearance. It may be inappropriate to include patients with incomplete LOS relaxation in the category of "hypertensive LOS". Such patients are better categorised as having an atypical disorder of LOS relaxation.

The value chosen for the lower limit of resting LOS pressure necessary to define a hypertensive LOS has ranged from $26.5^{46}$ to $45 \mathrm{~mm} \mathrm{Hg} .{ }^{47}$ A hypertensive LOS can be defined as one with a resting pressure value $>2 \mathrm{SD}$ above the normal mean value. Even by this definition however the lower limit will vary depending on the technique used to measure resting LOS pressure (rapid pull through versus station pull through; end expiratory, mid respiratory, end inspiratory). If mid respiratory LOS pressure is measured using the station pull through technique, a hypertensive LOS can be defined as one with a resting pressure of $>45 \mathrm{~mm} \mathrm{Hg}$. ${ }^{34} 48$

The manometric feature proposed for a diagnosis of isolated hypertensive LOS is a mean resting LOS pressure of $>45 \mathrm{~mm} \mathrm{Hg}$ measured in mid respiration using the station pull through technique. Patients who also have a mean distal oesophageal peristaltic wave amplitude $>180 \mathrm{~mm} \mathrm{Hg}$ are categorised as nutcracker oesophagus with a hypertensive LOS. Patients with incomplete LOS relaxation are categorised as having an atypical disorder of LOS relaxation, not as having an isolated hypertensive LOS.

\section{Oesophageal hypocontraction}

Scleroderma (progressive systemic sclerosis) has long been the paradigm disorder for oesophageal hypocontraction. Manometric evidence of oesophageal dysfunction can be found in approximately $80 \%$ of patients with scleroderma ${ }^{49}$ The underlying disease process appears to be one

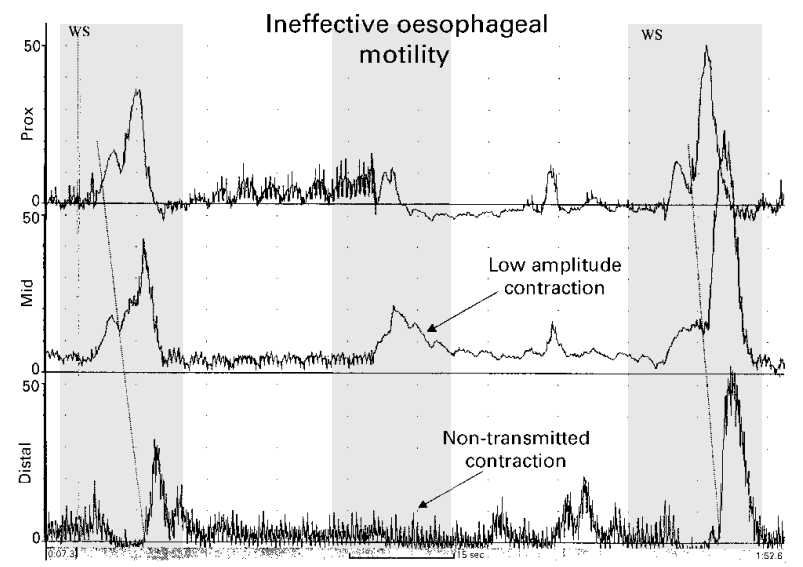

Figure 4 Oesophageal manometry tracing from a patient with ineffective oesophageal motility. The recording sites are positioned 3, 8, and $13 \mathrm{~cm}$ above the lower oesophageal sphincter. Note that the first and third wet swallows (WS) result in normal peristaltic sequences. However, the second wet swallow stimulates only low amplitude contractions in the proximal two leads, and no contraction in the distal lead (non-transmitted contraction or "failed peristalsis"). 
Table 3 Manometric features of named oesophageal motility abnormalities

\begin{tabular}{|c|c|c|c|c|}
\hline & Basal LOSP & LOS relaxation & Wave progression & Distal wave amplitude \\
\hline Achalasia & $\begin{array}{l}\text { Usually high, may be } \\
\text { normal, rarely low }\end{array}$ & Incomplete & Simultaneous or absent, no peristalsis & Low or normal \\
\hline $\begin{array}{l}\text { Atypical disorders of LOS } \\
\text { relaxation }\end{array}$ & Low, normal, or high & $\begin{array}{l}\text { Inadequate (incomplete } \\
\text { or short duration) }\end{array}$ & $\begin{array}{l}\text { Some normal peristalsis, may have } \\
\text { simultaneous or absent sequences }\end{array}$ & Low, normal, or high \\
\hline Isolated hypertensive LOS & High & Complete & Normal & Normal \\
\hline Diffuse oesophageal spasm & Low, normal, or high & Complete & Simultaneous in $>10 \%$ of swallows & Normal or high \\
\hline Nutcracker oesophagus & Low, normal, or high & Complete & Normal & High \\
\hline Ineffective oesophageal motility & Low or normal & Complete & Normal, simultaneous, or absent & Low in $\geqslant 30 \%$ of wet swallows \\
\hline
\end{tabular}

LOSP, lower oesophageal sphincter pressure; LOS, lower oesophageal sphincter.

of fibrosis and vascular obliteration that affects the oesophageal muscle and its innervation. ${ }^{50}$ This process weakens the muscles that comprise the LOS, thereby predisposing to gastro-oesophageal reflux. Muscle atrophy in the body of the oesophagus causes weak contractions. Studies have shown that oesophageal clearance is compromised significantly when the amplitude of peristaltic contractions in the distal oesophagus falls to values below $30 \mathrm{~mm} \mathrm{Hg} .^{51}{ }^{52}$ The combination of neural and muscular damage may result in loss of peristalsis. Scleroderma affects the smooth muscle of the distal oesophagus predominantly but the striated muscle of the proximal oesophagus may be involved in some cases. ${ }^{53}$

Although textbooks and review articles on scleroderma oesophagus frequently describe resting LOS pressures as very low $(<10 \mathrm{~mm} \mathrm{Hg}),{ }^{53}{ }^{54}$ recent studies suggest that mean LOS pressures are usually only moderately reduced in scleroderma. In a recent study of 36 patients with systemic sclerosis, for example, the mean resting LOS pressure (measured in mid respiration) was 15.8 (1.2) $\mathrm{mm}$ $\mathrm{Hg}$ in patients compared with 26.0 (2.1) $\mathrm{mm} \mathrm{Hg}$ in normal control subjects. ${ }^{55}$ If peristalsis is preserved, the peristaltic waves are often of low amplitude $(<30 \mathrm{~mm} \mathrm{Hg}) .^{54}{ }^{55}$ These low amplitude waves may involve the oesophagus in a patchy fashion. Abnormalities in the progression of peristalsis are observed frequently and include: (1) failed peristalsis in which the peristaltic wave progresses through the pharynx and proximal oesophagus but fails to traverse the entire length of the distal oesophagus, (2) simultaneous oesophageal contractions of low amplitude, and (3) absent oesophageal contractions (that is, swallowing results in no discernible contractions). ${ }^{51-56}$

The manometric features of scleroderma are by no means specific for this disorder. Identical manometric abnormalities can be found in patients with other collagen vascular disorders such as mixed connective tissue disease, rheumatoid arthritis, and systemic lupus erythematosus. ${ }^{57}$ Patients with certain non-rheumatic diseases such as diabetes mellitus, amyloidosis, alcoholism, myxoedema, and multiple sclerosis can also exhibit hypocontraction in the distal oesophagus indistinguishable from that of scleroderma, as can otherwise healthy patients who have gastrooesophageal reflux disease. ${ }^{57}$ Furthermore, in one study, patients with "scleroderma-like" oesophageal motility abnormalities who had no apparent rheumatic disease did not develop rheumatic disease over a follow up period of more than five years. ${ }^{57}$ For these reasons, use of the term "scleroderma oesophagus" is discouraged. If used at all, this term should be restricted only to patients who have scleroderma. The term "ineffective oesophageal motility" is preferable to describe patients with the constellation of findings typical of scleroderma.

Leite et al recently identified a group of 61 patients who had been diagnosed as having non-specific oesophageal motility disorders because their pattern of manometric abnormalities did not fall into any well defined category. ${ }^{58}$ A detailed review of this group showed that in 60 of 61 patients, the motility pattern was one of oesophageal hypocontraction that resulted in ineffective oesophageal motility. This phenomenon is observed commonly in patients with gastro-oesophageal reflux disease and is associated with poor oesophageal clearance of acid. As noted above, scleroderma is characterised by ineffective oesophageal motility. Although uncoordinated contractions of normal or high amplitude can also impair oesophageal motility and thus might be considered "ineffective", the term "ineffective oesophageal motility" is used to describe abnormalities characterised by hypocontraction.

The manometric features proposed for a diagnosis of ineffective oesophageal motility are evidence of hypocontraction in the distal oesophagus with at least $30 \%$ of wet swallows exhibiting any combination of the following abnormalities: (1) distal oesophageal peristaltic wave amplitude $<30 \mathrm{~mm} \mathrm{Hg}$ (fig 4), (2) simultaneous contractions with amplitudes $<30 \mathrm{~mm} \mathrm{Hg}$, (3) failed peristalsis in which the peristaltic wave does not traverse the entire length of the distal oesophagus (fig 4), or (4) absent peristalsis. Patients with ineffective oesophageal motility often have LOS hypotension. The LOS hypotension may not be severe however and studies suggest that abnormal oesophageal acid exposure in patients with ineffective oesophageal motility may correlate better with the abnormalities in peristaltic function than with resting LOS pressure. For these reasons, LOS hypotension is not required as a diagnostic criterion for ineffective oesophageal motility.

\section{Non-specific oesophageal motility abnormalities}

The conditions discussed above are those that have been recognised and named by investigators, and for which there are published series. The minimal requisite features for establishing a manometric diagnosis of the named oesophageal motility abnormalities are summarised in table 3. Many patients previously categorised as having "non-specific oesophageal motility disorders" would be included in the category of ineffective oesophageal motility. ${ }^{58}$ Nevertheless, one occasionally encounters patients who have oesophageal motility abnormalities with manometric features that do not meet the requisite criteria for any of these recognised conditions. Pending further advances in our understanding of the pathophysiology of motility abnormalities, non-specific oesophageal motility abnormalities should be reported descriptively.

Department of Medicine,

S J SPECHLER

Dallas VA Medical Center

and University of Texas Southwestern Medical Center at Dallas,

Dallas, Texas, USA

D O CASTELL

Department of Medicine,

Graduate Hospital, Philadelphia, Pennsylvania, USA

Correspondence to: Dr S J Spechler, Division of Gastroenterology (111B1), Dallas VA Medical Center, 4500 South Lancaster Road, Dallas, Texas 75216 , USA.sjspechler@aol.com

1 Alrakawi A, Clouse RE. The changing use of esophageal manometry in clinical practice. Am f Gastroenterol 1998;93:2319-20. 
2 Katz PO, Dalton CB, Richter JE, et al. Esophageal testing in patients with noncardiac chest pain or dysphagia: results of three years' experience with 987;106:593-7.

3 Cohen S. Esophageal motility disorders and their response to calcium channel antagonists. The sphinx revisited. Gastroenterology 1987;93:201-3.

4 Richter JE, Bradley LA, Castell DO. Esophageal chest pain: current controversies in pathogenesis, diagnosis, and therapy. Ann Intern Med 1989;110: 66-78.

5 Kahrilas PJ. Nutcracker esophagus: an idea whose time has gone? Am f Gastroenterol 1993;88:167-9.

6 Richter J, Dalton C, Bradley L, et al. Oral nifedipine in the treatment of noncardiac chest pain in patients with the nutcracker esophagus. Gastroenterology 1987;93:21-8.

7 Diamant NE. Regulation and dysregulation of esophageal motor function. In: Janssens J, ed. Progress in understanding and management of gastrointestinal motility disorders. Belgium: University of Leuven, 1993:85-103.

8 Spechler SJ, Souza RF, Rosenberg SJ, et al. Heartburn in patients with achalasia. Gut 1995;37:305-8.

9 Clouse RE, Staiano A. Manometric patterns using esophageal body and lower sphincter characteristics. Findings in 103 patients. Dig Dis Sci 1992; 37:289-96.

10 Kahrilas PJ, Dodds WJ, Hogan WJ. Effect of peristaltic dysfunction on esophageal volume clearance. Gastroenterology 1988;94:73-80.

11 Castell DO, Richter JE, Dalton CB, eds. Esophageal motility testing. New York: Elsevier Science Publishing Co., Inc., 1987.

12 Kahrilas PJ, Lin S, Chen J, et al. The effect of hiatus hernia on gastro-oesophageal junction pressure. Gut 1999;44:476-82.

13 Richter JE, Wu WC, Hohns DN, et al. Esophageal manometry in 95 healthy adult volunteers. Variability of pressures with age and frequency of "abnormal" contractions. Dig Dis Sci 1987;32:583-92.

14 Reynolds JC, Parkman HP. Achalasia. Gastroenterol Clin North Am 1989;18: 223-55.

15 Goldblum JR, Whyte RI, Orringer MB, et al. Achalasia. A morphologic study of 42 resected specimens. Am f Surg Pathol 1994;18:327-37.

16 Holloway RH, Dodds WJ, Helm JF, et al. Integrity of cholinergic innervation to the lower esophageal sphincter in achalasia. Gastroenterology 1986;90: 924-9.

17 Singaram C, Koch J, Gaumnitz EA, et al. Nature of neuronal loss in human achalasia. Gastroenterology 1996;110:A259.

18 Saha HK, Sengupta JN, Goyal RK. Role of chloride ions in lower esophageal sphincter tone and relaxation. Am F Physiol 1992;263:G115-26.

19 Yamato S, Spechler SJ, Goyal RK. Role of nitric oxide in esophageal peristalsis in the opossum. Gastroenterology 1992;103:197-204.

20 Goldenberg SP, Burrell M, Fette GC, et al. Classic and vigorous achalasia: a comparison of manometric, radiographic, and clinical findings. Gastroenterology 1991;101:743-8.

21 De Oliveira RB, Rezende Filho J, Dantas RO, et al. The spectrum of esophageal motor disorders in Chagas' disease. Am f Gastroenterol 1995;90: $1119-24$.

22 Kahrilas PJ, Kishk SM, Helm JF, et al. Comparison of pseudoachalasia and achalasia. Am ₹ Med 1987;82:439-46.

23 Spechler SJ. AGA technical review on treatment of patients with dysphagia caused by benign disorders of the distal esophagus. Gastroenterology 1999; 117:233-54.

24 Vantrappen G, Janssens J, Hellemans J, et al. Achalasia, diffuse esophageal spasm, and related motility disorders. Gastroenterology 1979;76:450-7.

25 Goldblum JR, Rice TW, Richter JE. Histopathologic features in esophagomyotomy specimens from patients with achalasia. Gastroenterology 1996; 111:648-54.

26 Katz PO, Richter JE, Cowan R, et al. Apparent complete lower esophageal sphincter relaxation in achalasia. Gastroenterology 1986;90:978-83.

27 Dudnick RS, Castell JA, Castell DO. Abnormal upper esophageal sphincter function in achalasia. Am f Gastroenterol 1992;87:1712-15.

28 Zhang ZG, Diamant NE. Repetitive contractions of the upper esophageal body and sphincter in achalasia. Dysphagia 1994;9:12-19.

29 Massey BT, Hogan WJ, Dodds WJ, et al. Alteration of the upper esophageal sphincter belch reflex in patients with achalasia. Gastroenterology 1992;103: 1574-9.

30 Pasricha PJ, Rai R, Ravich WJ, et al. Botulinum toxin for achalasia: long-term outcome and predictors of response. Gastroenterology 1996;110: $1410-15$
31 Cuilliere C, Ducrotte P, Zerbib F, et al. Achalasia: outcome of patients treated with intrasphincteric injection of botulinum toxin. Gut 1997;41:8792

32 Richter JE, Castell DO. Diffuse esophageal spasm: a reappraisal. Ann Intern Med 1984;100:242-5.

33 Dalton CB, Castell DO, Hewson EG, et al. Diffuse esophageal spasm. A rare motility disorder not characterized by high-amplitude contractions. Dig Dis Sci $1991 ; 36: 1025-8$.

34 Achem SR, Benjamin SB. Esophageal dysmotility (spastic dysmotility). In: Castell DO, ed. The esophagus, 2nd edn. Boston: Little, Brown and Company, 1995:247-68.

35 Allen ML, DiMarino AJ. Manometric diagnosis of diffuse esophageal spasm. Dig Dis Sci 1996;41:1346-9.

36 Dalton CB, Castell DO, Richter JE. The changing faces of the nutcracker esophagus. Am $\mathcal{F}$ Gastroenterol 1988;83:623-8.

37 Benjamin SB, Gerhardt DC, Castell DO. High amplitude, peristaltic esophageal contractions associated with chest pain and/or dysphagia. Gastroenterology $1979 ; 77: 478-83$.

38 Clouse RE, Staiano A. Topography of normal and high-amplitude esophageal peristalsis. Am f Physiol 1993;265:G1098-107.

39 Achem SR, Kolts BE, Burton L. Segmental versus diffuse nutcracker esophagus: an intermittent motility pattern. Am f Gastroenterol 1993;88: $847-51$.

40 Freidin N, Mittal RK, Traube M, et al. Segmental high amplitude peristaltic contractions in the distal esophagus. Am F Gastroenterol 1989;84:619-23.

41 Herrington JP, Burns TW, Balart LA. Chest pain and dysphagia in patients with prolonged peristaltic contractile duration of the esophagus. Dig Dis Sci 1984;29:134-40.

42 Code CF, Schlegel JF, Kelley ML, et al. Hypertensive gastroesophageal sphincter. Proc Mayo Clin 1960;35:391-9.

43 Bassotti G, Alunni G, Cocchieri M, et al. Isolated hypertensive lower esophageal sphincter. Clinical and manometric aspects of an uncommon esophageal motor abnormality. F Clin Gastroenterol 1992;14:285-7.

44 Garrett JM, Godwin DH. Gastroesophageal hypercontracting sphincter. Manometric and clinical characteristics. $\mathscr{F} A M A$ 1969;208:992-8.

45 Freidin N, Traube M, Mittal RK, et al. The hypertensive lower esophageal sphincter. Manometric and clinical aspects. Dig Dis Sci 1989;34:1063-7.

46 Katada N, Hinder RA, Hinder PR, et al. The hypertensive lower esophageal sphincter. Am f Surg 1996;172:439-43.

47 Katzka DA, Sidhu M, Castell DO. Hypertensive lower esophageal sphincter pressures and gastroesophageal reflux: an apparent paradox that is not unusual. Am $\mathcal{F}$ Gastroenterol 1995;90:280-4.

48 Waterman DC, Dalton CB, Ott DJ, et al. Hypertensive lower esophageal sphincter: what does it mean? f Clin Gastroenterol 1989;11:139-46.

49 Bassotti G, Battaglia E, Debernardi V, et al. Esophageal dysfunction in scleroderma. Relationship with disease subsets. Arthritis Rheum 1997;40:22529 .

50 Lock G, Holstege A, Lang B, et al. Gastrointestinal manifestations of progressive systemic sclerosis. Am f Gastroenterol 1997;92:763-71.

51 Richter JE, Blackwell JN, Wu WC, et al. Relationship of radionuclide liquid bolus transport and esophageal manometry. F Lab Clin Med 1987;109:21724 .

52 Turner R, Lipshutz W, Miller W, et al. Esophageal dysfunction in collagen disease. Am f Med Sci 1973;265:191-9.

53 Cohen S, Laufer I, Snape WJ Jr, et al. The gastrointestinal manifestations of scleroderma: pathogenesis and management. Gastroenterology 1980;79: $155-66$.

54 Cohen S. Motor disorders of the esophagus. N Engl F Med 1979;301:18492.

55 Yarze JC, Varga J, Stampfl D, et al. Esophageal function in systemic sclerosis: a prospective evaluation of motility and acid reflux in 36 patients. Am $\mathcal{f}$ Gastroenterol 1993;88:870-6.

56 Zamost BJ, Hirschberg J, Ippoliti AF, et al. Esophagitis in scleroderma. Prevalence and risk factors. Gastroenterology 1987;92:421-8.

57 Schneider HA, Yonker RA, Longley S, et al. Scleroderma esophagus: a nonspecific entity. Ann Intern Med 1984;100:848-50.

58 Leite LP, Johnston BT, Barrett J, et al. Ineffective esophageal motility (IEM). The primary finding in patients with nonspecific esophageal motility disorder. Dig Dis Sci 1997;42:1859-65. 